\title{
Phenomenological model for coupled multi-axial piezoelectricity
}

\section{Yuchen Wei, Sergio Pellegrino}

Yuchen Wei, Sergio Pellegrino, "Phenomenological model for coupled multiaxial piezoelectricity," Proc. SPIE 10596, Behavior and Mechanics of Multifunctional Materials and Composites XII, 105960R (22 March 2018); doi: $10.1117 / 12.2296346$

EDIE SPIE Smart Structures and Materials + Nondestructive Evaluation and Health Monitoring, 2018, Denver, Colorado, United States 


\title{
Phenomenological Model for Coupled Multi-axial Piezoelectricity
}

\author{
Yuchen Wei and Sergio Pellegrino \\ California Institute of Technology, 1200 E California Blvd, Pasadena, USA
}

\begin{abstract}
A quantitative calibration of an existing phenomenological model for polycrystalline ferroelectric ceramics is presented. The model relies on remnant strain and polarization as independent variables. Innovative experimental and numerical model identification procedures are developed for the characterization of the coupled electro-mechanical, multi-axial nonlinear constitutive law. Experiments were conducted on thin PZT-5A4E plates subjected to cross-thickness electric field. Unimorph structures with different thickness ratios between PZT-5A4E plate and substrate were tested, to subject the piezo plates to coupled electro-mechanical fields. Material state histories in electric field-strain-polarization space and stress-strain-polarization space were recorded. An optimization procedure is employed for the determination of the model parameters, and the calibrated constitutive law predicts both the uncoupled and coupled experimental observations accurately.
\end{abstract}

Keywords: Ferroelectrics, phenomenological model, parameter calibration, electro-mechanical coupling

\section{INTRODUCTION}

Ferroelectric materials exhibit nonlinear behavior under strong electric fields, stress fields, or a combination of the two. The nonlinearity is more evident in polycrystalline ferroelectric materials due to the evolution of complex domain structures which is also the source of large electro-mechanical coupling coefficients. Such unique properties combined with the large blocking force capability, make ferroelectric-material-based devices favorable in active structure applications that require both high precision and large stroke. Ferroelectric material based unimorph and bimorph structures have been widely used in MEMS applications (Fleming ${ }^{1}$, Rahman ${ }^{2}$ ) for displacement control and large scale adaptive optics, ${ }^{4}$ just to name a few. A comprehensive introduction can be found in monographs, such as Ref. 3. Design and operation of these active structures posed two main challenges: tracking the material state evolution under complex loading conditions (both electrical and mechanical) to guarantee precise operation; and optimal design of the structures to obtain a desired response under actuation which is key to a successful design. The paper aims to provide tools to tackle both challenges by calibrating a macroscopic nonlinear material model ${ }^{18}$ and incorporate it into efficient estimation algorithms for active unimorph structures, and then validate against experimental measurements.

There has been a continuous pursuit of ferroelectric material models that can capture domain switching induced material nonlinearity. These models can be broadly categorized into microscopic and macroscopic approaches, depending on how the model relate the macroscopic level properties and responses to the microscopic domain structure evolution. An overview of various modeling approaches to date can be found in Landis, ${ }^{6}$ Potnis, ${ }^{7}$ and Huber. ${ }^{8,9}$ The mainstream method among microscopic approaches is the phase field method, which implements a diffuse-interface modeling framework to track the evolution of ferroelectric domains. Representative works were presented by Landis, ${ }^{10}$ Schrade $^{11}$ and Vidyasagar. ${ }^{12}$ Compatibility at the domain boundary among different crystal variants and numerical stability and convergence issues pose critical challenges. In the present paper, due to its simplicity and robustness, a macroscopic phenomenological model for polycrystalline ferroelectric materials is chosen. The macroscopic approach was initiated from the work by Hwang ${ }^{13}$ and Lynch. ${ }^{14}$ The modeling approach is mathematically sound, generally established within the formalism of thermodynamic framework. The constitutive law is established based on postulated Helmholtz free energy and hypothesized switching function defined in internal variable space. Computational efficiency is one of the major advantages

Yuchen Wei: E-mail: ywei@caltech.edu

Sergio Pellegrino: E-mail: sergiop@caltech.edu, Telephone: +1 6263954764

Behavior and Mechanics of Multifunctional Materials and Composites XII,

edited by Hani E. Naguib, Proc. of SPIE Vol. 10596, 105960R - ( 2018 SPIE

CCC code: $0277-786 X / 18 / \$ 18 \cdot$ doi: $10.1117 / 12.2296346$

Proc. of SPIE Vol. 10596 105960R-1 
of these models due to the reduced number of unknown parameters; however at the cost that calculations reveal no information on the micro-structure evolution is described. Representative publications include Cocks, ${ }^{15}$ Kamlah, ${ }^{16,17}$ Landis, ${ }^{18-20}$ Klinkel, ${ }^{21,22}$ Maniprakash. ${ }^{23,24}$ This paper mainly follows the framework proposed by Landis. ${ }^{18}$

The remainder of the paper is organized as follows. Section 2 briefly reviews the full field, multi-axial constitutive law proposed in Landis ${ }^{18}$ which is then incorporated in a classical lamination theory based estimation model for actuation response. An implicit, backward Euler based integration algorithm for the constitutive law is then briefly described. Section 3 describes experimental procedures to calibrate the model parameters, where PZT-5A thin sheets were subjected to electrical and mechanical stress loading, both separately and in combination. Detailed results and discussions of the model parameter identification process are presented in section 4. Among different model parameters fitted from different loading conditions, an optimal set of parameters was chosen which yielded the least relative error with respect to the whole experimental measurement set. Section 5 concludes the paper.

\section{DESCRIPTION OF CONSTITUTIVE MODEL AND IMPLEMENTATION IN ACTIVE STRUCTURES}

\subsection{Overview of fully coupled multi-axial constitutive law}

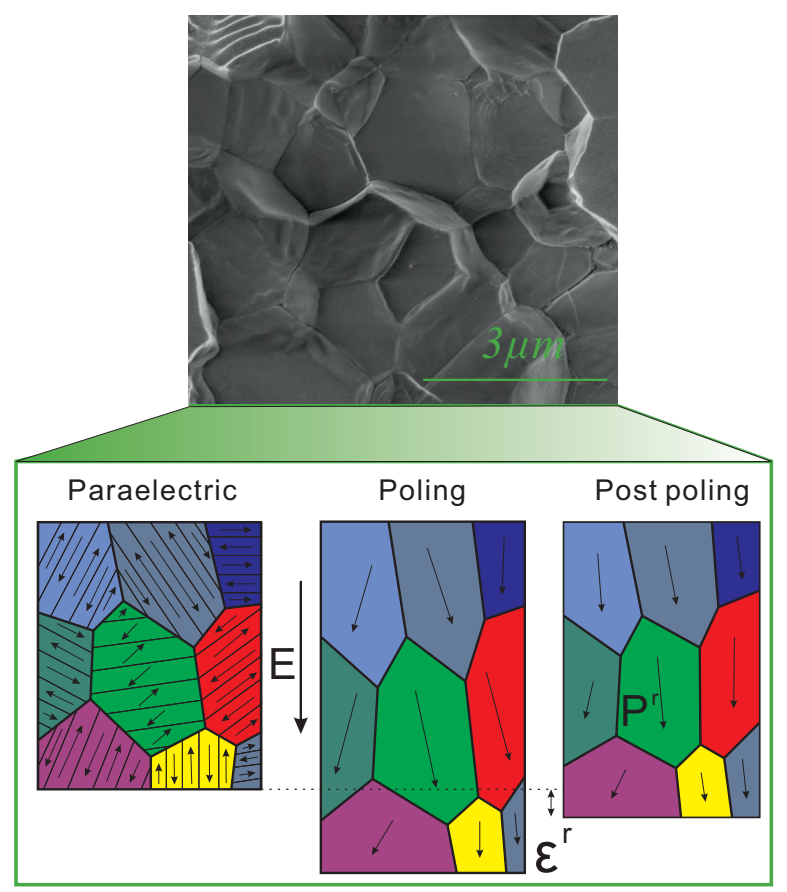

Figure 1. Illustration of internal variables in phenomenological model for polycrystalline ferrorelectric ceramics. Top figure is a Scanning Electron Microscope (SEM) image of the surface of a PZT-5A plate, average grain size $\sim 5 \mu m$. After subjecting the material to a strong electric field, material developed a macroscopic initial polarization and strain. Internal variables $\varepsilon^{r}$ and $P^{r}$ can be viewed as volumetric average of the polarization and strain over the entire domain.

The nonlinear constitutive law of piezoelectric material is given by equation $1:^{18}$

$$
\begin{aligned}
D_{m} & =d_{m k l} \sigma_{k l}+\kappa_{m n} \mathcal{E}_{n}+P_{m}^{r} \\
\varepsilon_{i j} & =s_{i j k l} \sigma_{k l}+d_{n i j} \mathcal{E}_{n}+\varepsilon_{i j}^{r}
\end{aligned}
$$

Here $D_{m}$ is the electric displacement vector, $\varepsilon_{i j}$ the total strain tensor, $d_{m k l}$ the electro-mechanical coupling coefficient, $\sigma_{k l}$ the mechanical stress tensor, $\kappa_{m n}$ the dielectric coefficient tensor, $P_{m}$ the remnant polarization vector, $s_{i j k l}$ the compliance tensor, $\varepsilon_{i j}^{r}$ the remnant strain tensor. In this paper $\alpha, \beta \in\{1,2\}$ and $i, j, k, l \in$ 
$\{1,2,3\} \cdot \varepsilon_{i j}^{r}$ and $P_{m}^{r}$ are independent state variables, shown in figure 1 . The core of the framework in $\operatorname{Ref}^{18}$ is composed of functional forms of Helmholtz free energy of electro-mechanical coupling $\Psi$ and switching function $\Phi$. The switching surface, within which the material displays no ferroelectric switching, ${ }^{8}$ takes the following form:

$$
\Phi=\frac{3 \hat{S}_{i j} \hat{S}_{i j}}{2 \sigma_{0}^{2}}+\frac{\hat{\mathcal{E}}_{i} \hat{\mathcal{E}}_{i}}{\mathcal{E}_{0}^{2}}+\frac{\beta \hat{\mathcal{E}}_{i} P_{j}^{r} \hat{S}_{i j}}{\mathcal{E}_{0} P_{0} \sigma_{0}}-1=0
$$

$\mathcal{E}_{0}$ is the ferroelectric coercive field, and $\sigma_{0}$ the stress field when ferroelastic switching started for uniaxial tension or compression. The effective electric field $\hat{\mathcal{E}}$ and effective deviatoric stress field $\hat{S}$ are defined as follows:

$$
\begin{aligned}
\hat{\mathcal{E}}_{i} & =\mathcal{E}_{i}-\mathcal{E}_{i}^{B} \\
\hat{S_{i j}} & =S_{i j}-S_{i j}^{B}
\end{aligned}
$$

$\mathcal{E}$ and $S$ are external electric and deviatoric stress fields. Back stress field $S_{i j}^{B}$ and back electric field $\mathcal{E}_{i}^{B}$ represent constraints during domain evolution due to surrounding lattice structures, obtained by differentiation of the potential functions:

$$
\sigma_{i j}^{B}=\frac{\partial \Psi^{r}}{\partial \epsilon_{i j}^{r}}, \mathcal{E}_{i}^{B}=\frac{\partial \Psi^{r}}{\partial P_{i}^{r}}
$$

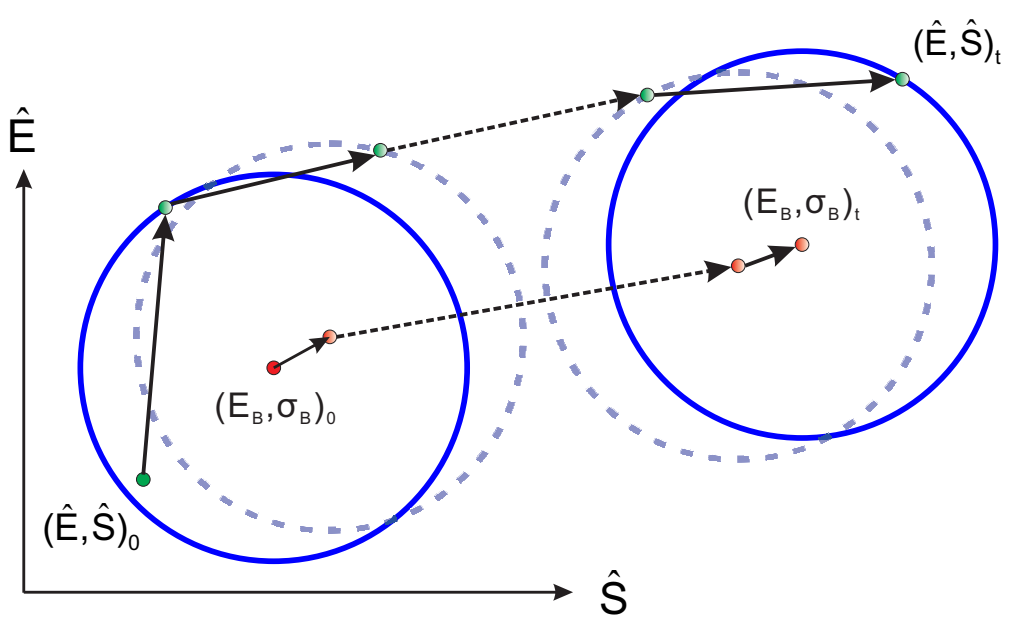

Figure 2. Movement of yield surface during kinematic hardening of material in generalized stress - electric field space. Red dots represent the center of yield surface, determined by current internal variables; green dots represent the external fields history; dotted circles represent intermediate steps during hardening.

The incremental plasticity-like formulation of the switching process is visualized in figure 2. Evolution of material micro structure is modeled as shifts of center position of yield surface (red dots), once the material state is driven to the yield surface $(\Psi=0)$. Reference ${ }^{20}$ proposed functional forms of potential $\Psi^{r}$ based on a semiheuristic approximation procedure, which can be decomposed into $\Psi^{\sigma}$ and $\Psi^{E}$. In total 11 model parameters need to be calibrated which describe all the functional forms implemented. Details about the parameter definitions are explained in Ref. ${ }^{18}$ Calibration of the parameters involves comparing estimations and measurements of $D$ and $\varepsilon$ under external fields $\mathcal{E}$ and $\sigma$. Details will be presented in section 4.

\subsection{Active structure model and integration of constitutive law}

We have developed an efficient implementation of the phenomenological model reviewed in Section 2 and integrated it with an actuation model for piezoceramics bonded to thin shell structures. The general approach treats the actuation response of such active structures as a thermal expansion problem, first presented by Crawley. ${ }^{26}$ Models of this kind have been established to study the optimal design of these structures and were widely validated by experiments, ${ }^{28,31,32}$ but mainly linear material properties were assumed. We take a similar approach here. We start by considering thin, layered structures composed of laminated active/nonactive layers, based on 
the assumption that each layer is mechanically isotropic or orthotropic in-plane. Following a derivation similar to classical lamination theory for composite laminates in Ref 27 , the governing equation of active laminated structures have the form:

$$
\left[\begin{array}{c}
\mathbf{F} \\
\mathbf{M}
\end{array}\right]=\left[\begin{array}{ll}
\mathbf{A} & \mathbf{B} \\
\mathbf{B} & \mathbf{D}
\end{array}\right]\left[\begin{array}{c}
\varepsilon \\
\mathbf{K}
\end{array}\right]-\left[\begin{array}{c}
\mathbf{F}_{\Lambda} \\
\mathbf{M}_{\Lambda}
\end{array}\right]
$$

Here $\mathbf{F}$ and $\mathbf{M}$ are external force and moment vectors of stress resultants, $\boldsymbol{\varepsilon}$ and $\mathbf{K}$ are in-plane strain and curvature of the mid-plane of the structure, and $\mathbf{F}_{\Lambda}, \mathbf{M}_{\Lambda}$ are actuation vectors. To incorporate the phenomenological constitutive law, the forcing terms are both history and internal variables dependent, i.e.:

$$
\left[\begin{array}{c}
\mathbf{F}_{\Lambda} \\
\mathbf{M}_{\Lambda}
\end{array}\right]=\left[\begin{array}{c}
\mathbf{F}_{\Lambda}\left(\sigma, \mathcal{E}, \mathbf{P}^{r}, \varepsilon^{r}\right) \\
\mathbf{M}_{\Lambda}\left(\sigma, \mathcal{E}, \mathbf{P}^{r}, \varepsilon^{r}\right)
\end{array}\right]
$$

The stress and electric fields $\sigma$ and $\mathcal{E}$, together, determine the internal variables of the ferroelectric layer. Thus both the actuation vectors and the response $\varepsilon$ and $\mathbf{K}$ must be solved iteratively at each loading step, and the constitutive law has to been integrated along the loading path. In this work, the constitutive law is integrated using a backward Euler, fully implicit scheme, inspired by Belytschko. ${ }^{35}$ Derivations were carried out with the symbolic computation software Mathematica ${ }^{\circledR}$.

\section{EXPERIMENTAL MEASUREMENTS}

This section describes the experimental methodologies which provided measurement data sets for model parameter calibration. All measurements were conducted on commercially available lead zirconate-titanate PZT-5A4E (Industry type 5A, Navy type II) thin sheets from Piezo Systems Inc ${ }^{\circledR}$, and unimorph structures manufactured by bonding the PZT-5A4E sheets to thin aluminum substrates. The PZT-5A4E sheet is referred as piezoelectric sheet in the rest of the paper. Its composition belongs to a standard 'soft' type ferroelectric material, and its crystal structure is at the morphotropic phase boundary in the composition-temperature phase diagram. This unique composition exploits the transitions between tetragonal, rhombohedral and intermediary phases to achieve large dielectric permittivity and piezoelectric effects ${ }^{29}$ meanwhile endows nonlinearity such as dielectric hysteresis to the material. Within the characterization experiments, two types of external fields were applied: a pure electric field, and the combination of electric and mechanical fields, to invoke both ferroelectric and ferroelastic switching in the material. Results of the measurements and discussions will be presented in section 4 together with model calibrations.

\subsection{Ferroelectric switching tests}

In the first set of tests, the electric field $\mathcal{E}$ exclusively drives the domain evolution of a free standing piezoelectric sheet. During these experiments a strong, cyclic electric field was applied parallel to the poling direction with the following form:

$$
\mathcal{E}_{3}=\mathcal{E}_{0} \sin \left(\frac{2 \pi t}{T}\right)
$$

Subscripts 1, 2 denote in-plane direction of the piezoelectric sheet, and 3 denotes the thickness direction. Material response in this case can be estimated as:

$$
\begin{aligned}
D_{3} & =\kappa_{3} \mathcal{E}_{3}+P_{3}^{r} \\
\varepsilon_{\alpha} & =d_{3 \alpha} \mathcal{E}_{3}+\varepsilon_{\alpha}^{r}
\end{aligned}
$$

$D_{3}$ and $\varepsilon_{\alpha}$ are measured experimentally. Piezoelectric sheets of two thicknesses: $127 \mu m$ and $200 \mu m$ were tested. The plates were pre-poled along the thickness direction, and had a manufactured dimensions of $7 \mathrm{~cm} \times 7 \mathrm{~cm}$; the top and bottom surfaces were coated with thin Nickel electrodes. A schematic of experiment is shown in figure 3 . Driving field signal was generated using an Agilent 33210A function generator, and amplified by a Trek ${ }^{\circledR}$ model 10/10B-HS high voltage amplifier. A Sawyer-Tower type circuit ${ }^{30}$ was used to measure the electric displacement $D_{3}$ of the actuated plates. A $10 \mu F$ sensing capacitor was used in the circuit, and its voltage was measured with an oscilloscope. The Correlated-Solution ${ }^{\circledR}$ Digital Image Correlation (DIC) system was used to record the in-plane strain $\varepsilon_{\alpha}$ during actuation. 


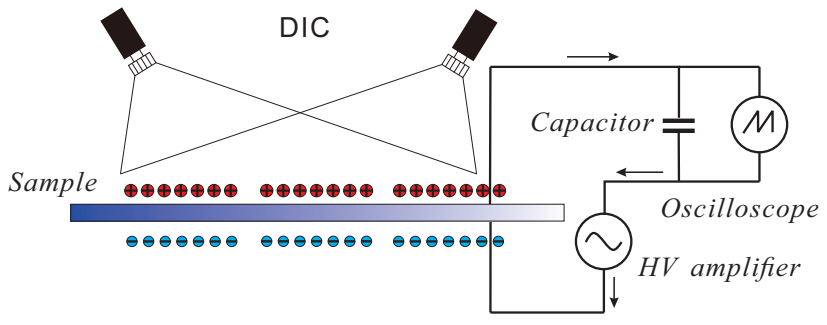

(a)

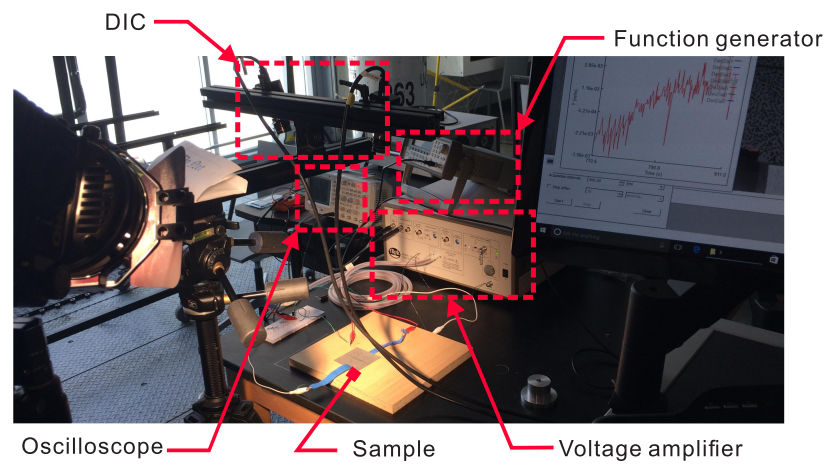

(b)

Figure 3. Ferroelectric switching test illustration and measurement setup.

\subsection{Unimorph actuation}

The following two sets of measurements focused on the response of piezoelectric sheets bonded to unimorph structures, when electric and stress fields co-exist which represents the most common case in active structures applications. In these tests, piezo sheets bonded unimorphs with different thickness properties were actuated under through-thickness electric fields. Two types of unimorph structures were tested, in the forms of strip and plate samples, as shown in figure 4. For strip samples, plane strain condition is assumed for $1-3$ plane; for plate samples, plane stress condition is assumed for both $1-3$ and $2-3$ planes. The ferroelectric material response can be estimated as:

$$
\begin{aligned}
D_{3} & =d_{3 \alpha} \sigma_{\alpha}+\kappa_{3} \mathcal{E}_{3}+P_{3}^{r} \\
\varepsilon_{\alpha} & =s_{\alpha \beta} \sigma_{\beta}+d_{3 \alpha} \mathcal{E}_{3}+\varepsilon_{\alpha}
\end{aligned}
$$

In-plane strain $\varepsilon_{\alpha}$ and curvature $\kappa$ of the piezo sheets are obtained from the experiments.

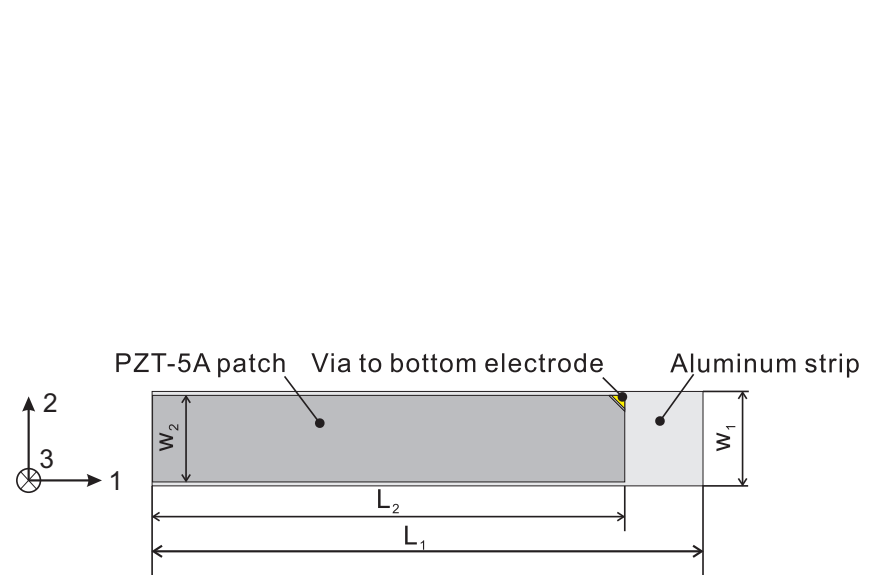

(a) Strip unimorph

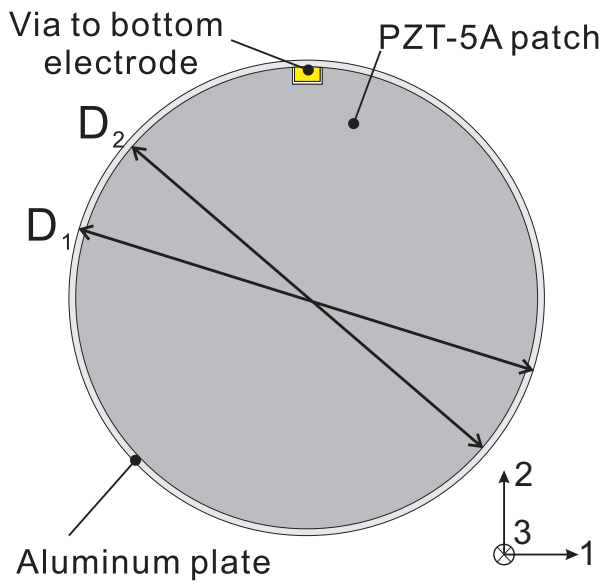

(b) Plate unimorph

Figure 4. Top view of sample geometries for strip and plate unimorph samples, corresponds to plane strain and plane stress conditions for the piezoelectric layer.

Sample geometries are listed as follows:

- Strip unimorph: $L_{1}=70 \mathrm{~mm}, L_{2}=60 \mathrm{~mm}, W_{1}=12 \mathrm{~mm}, L_{2}=11.8 \mathrm{~mm}$.

- Plate unimorph: $D_{1}=71 \mathrm{~mm}, D_{2}=70 \mathrm{~mm}$. 
Define the thickness ratio $R$ as:

$$
R=\frac{t_{a}}{t_{s}}
$$

From section 2, the electric field generated piezoelectric strain will induce mechanical stress components $\sigma_{11}$ and $\sigma_{22}$, which depends on $\mathcal{E}, R$ and the sample geometry. Five different thickness ratios were chosen, with the detailed dimensions listed in table 1.

Table 1. Sample thickness properties for actuation tests

\begin{tabular}{cccc}
\hline \hline Sample set \# & $\begin{array}{c}\text { Piezoelectric } \\
\text { layer }(\mu \mathrm{m})\end{array}$ & $\begin{array}{c}\text { Substrate } \\
\text { layer }(\mu \mathrm{m})\end{array}$ & $R$ \\
\hline 1 & 127 & 400 & 0.3175 \\
2 & 127 & 500 & 0.254 \\
3 & 127 & 600 & 0.212 \\
4 & 200 & 400 & 0.5 \\
5 & 200 & 500 & 0.4 \\
6 & 200 & 600 & 0.33 \\
\hline
\end{tabular}

The thicknesses of the epoxy bonding layer $t_{e}$ of the tested samples were also measured, and taken into account in the actuation model. Due to the different bonding techniques, for strip unimorphs $t_{e} \approx 30 \mu m$; for plate unimorphs $t_{e} \approx 100 \mu \mathrm{m}$. Two types of electric signals were used to actuate the piezoelectric layers for all samples: incremental step-wise actuation up to $1.25 \mathrm{MV} / \mathrm{m}$ with an interval of $0.25 \mathrm{MV} / \mathrm{m}$, and linear triangular wave with peak magnitudes of $1 \mathrm{MV} / \mathrm{m}, 2 \mathrm{MV} / \mathrm{m}$ or $3 \mathrm{MV} / \mathrm{m}$. Experiments were carried with the same equipment as in section 3.1.

\section{EXPERIMENT RESULTS AND MODEL CALIBRATION}

In this section a systematic calibration of model parameters in the constitutive law is described, and a comparison between fitted model and measurements is presented. All experimental data were fed into the calibration scheme described in section 4.1. The model parameters were fitted by minimizing the RMS error between macroscopic measurements of material history (strain and electric displacement) and estimations. The fitting was carried out according to the following sequence: each measurement set was fitted independently first, then the complete set was fitted. Different fitted parameters were used to cross check the RMS error under different loading conditions. An optimal parameter set was identified which yielded the minimum RMS error over the total measurement data.

\subsection{Calibration scheme}

We defined the parameter fitting process as the following optimization problem. Consider the normalized rootmean-square deviation (NRMSD) error between estimations and experimental measurements:

$$
\begin{aligned}
\min _{\mathcal{P}} N R M S D & =w_{\varepsilon} \cdot \sqrt{\frac{\sum_{i}^{N_{\varepsilon}}\left(\varepsilon_{\text {measured }}^{i}-\varepsilon_{\text {estimated }}^{i}\right)^{2}}{N_{\varepsilon} \varepsilon_{\text {amp }}}} \\
& +w_{K} \cdot \sqrt{\frac{\sum_{i}^{N_{K}}\left(K_{\text {measured }}^{i}-K_{\text {estimated }}^{i}\right)^{2}}{N_{K} K_{\text {amp }}}}
\end{aligned}
$$

The total error $\mathcal{E}$ is the sum of estimation deviation of (structural) curvature response, active layer strain measurements and electric displacement response. $N_{\varepsilon}$ and $N_{K}$ are the numbers of sample points under each measurement category, $\varepsilon_{a m p}$ and $K_{a m p}$ are maximum absolute value of each type of measurement. $w_{\varepsilon}$ and $w_{K}$ 
are weight constants for the error terms corresponding to the two types of experiments. $w_{\varepsilon}$ and $w_{K}$ take the values 1 and 0 alternatively in the optimizations in section 4.2 and section 4.3 ; they are both assigned the value 0.5 in section 4.4. The optimization problem was solved in Matlab using the unconstrained routine fminsearch.

\subsection{Ferroelectric calibration}

Under a pure electric field, the evolution of the material state is dominated by ferroelectric domain movement. Correspondingly, in $\hat{\mathcal{E}}-\hat{S}$ space, mainly the second term of the switching function determines the current position of the switching surface:

$$
\frac{3 \hat{S}_{i j} \hat{S}_{i j}}{2 \sigma_{0}^{2}}+\underbrace{\frac{\hat{\mathcal{E}}_{i} \hat{\mathcal{E}}_{i}}{\mathcal{E}_{0}^{2}}}_{\text {Term II }}+\frac{\beta \hat{\mathcal{E}}_{i} P_{j}^{r} \hat{S}_{i j}}{\mathcal{E}_{0} P_{0} \sigma_{0}}-1=0
$$

The parameters that strongly correlate with material response in the fitting process are the ones related to ferroelectric potential function $\Psi^{E}$. The coercive fields $E_{0}$ can be determined from the butterfly curve directly, and is kept constant during parameter update.

The results of a calibration and a comparison with experiment data are shown in figure 5. Calibrated model has achieved good agreement with both $\varepsilon$ and $D$ measurements. However the tests revealed a more abrupt transition around $\mathcal{E}_{0}$, which is not captured by the model.

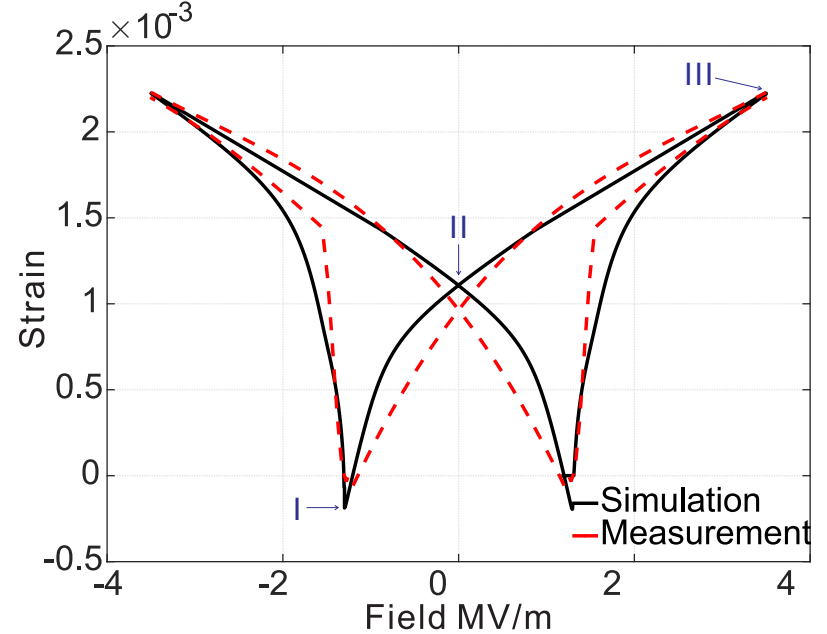

(a)

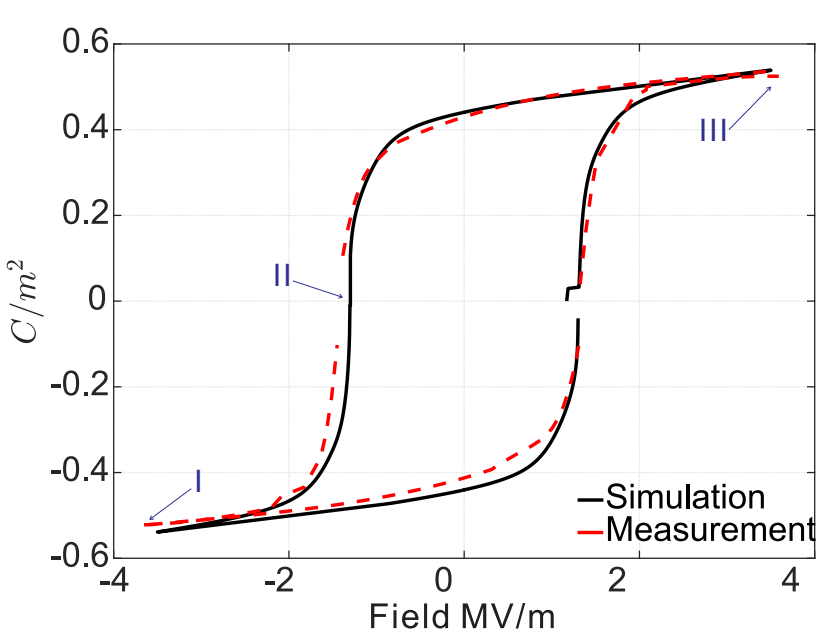

(b)

Figure 5. Ferroelectric experiments and fitting results. (a) Strain butterfly curve. $E_{0}=1.3 \mathrm{MV} / \mathrm{m}$ can be determined directly. (b) Dielectric hysteresis curve.

\subsection{Unimorph calibration}

In unimorph actuation tests, the electric field and the induced stress field jointly determine the current material state in $\hat{\mathcal{E}}-\hat{S}$ space. Thus at every new loading step, all terms in switching function will contribute to hardening calculation during material switching:

$$
\underbrace{\frac{3 \hat{S}_{i j} \hat{S}_{i j}}{2 \sigma_{0}^{2}}}_{\text {Term I }}+\underbrace{\frac{\hat{\mathcal{E}}_{i} \hat{\mathcal{E}}_{i}}{\mathcal{E}_{0}^{2}}}_{\text {Term II }}+\underbrace{\frac{\beta \hat{\mathcal{E}}_{i} P_{j}^{r} \hat{S}_{i j}}{\mathcal{E}_{0} P_{0} \sigma_{0}}}_{\text {Term III }}-1=0
$$

Since the stress field cannot be obtained directly, it has to be estimated from the measured variables. In the absence of mechanical loading, the following relation holds (from equation 5):

$$
\left[\begin{array}{c}
\varepsilon \\
K
\end{array}\right]=\left[\begin{array}{c}
\mathbf{F} \\
\mathbf{M}
\end{array}\right]\left[\begin{array}{ll}
\mathbf{A} & \mathbf{B} \\
\mathbf{B} & \mathbf{D}
\end{array}\right]^{-1}\left[\begin{array}{c}
\mathbf{F}_{\Lambda} \\
\mathbf{M}_{\Lambda}
\end{array}\right]
$$


The stress field is then estimated as:

$$
\sigma_{i j}=s_{i j k l}^{-1}\left(\varepsilon_{i j}-d_{n i j} \mathcal{E}_{n}-\varepsilon_{i j}^{r}\right)
$$

and was calculated at each integration step. Calibrations were performed on plane strain and plane stress actuation tests separately. Experimental measurements and calibrated model estimations for plane strain and plane stress conditions are shown in figure 6,7 , and figure 8,9 , for different thickness ratios $R$.

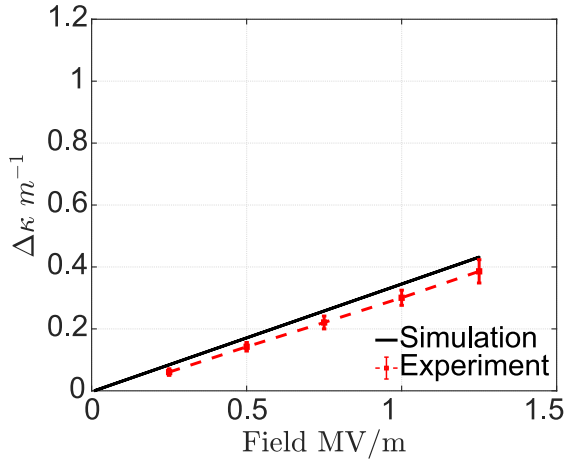

(a) $R=0.212$

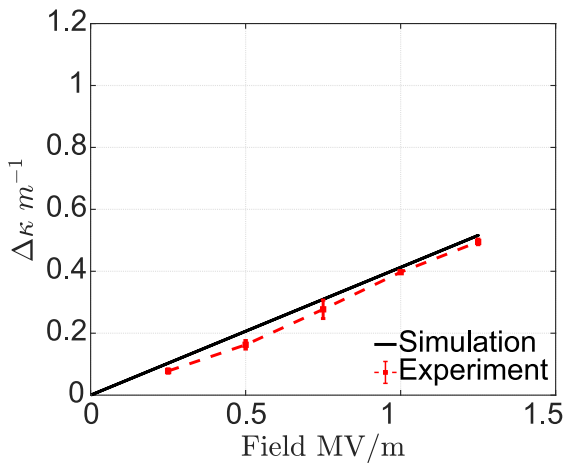

(d) $R=0.33$

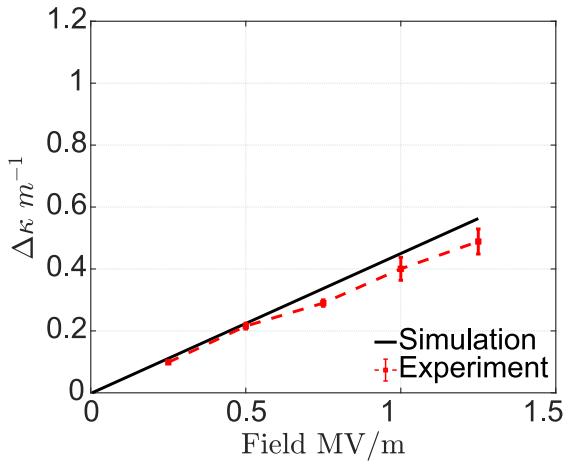

(b) $R=0.254$

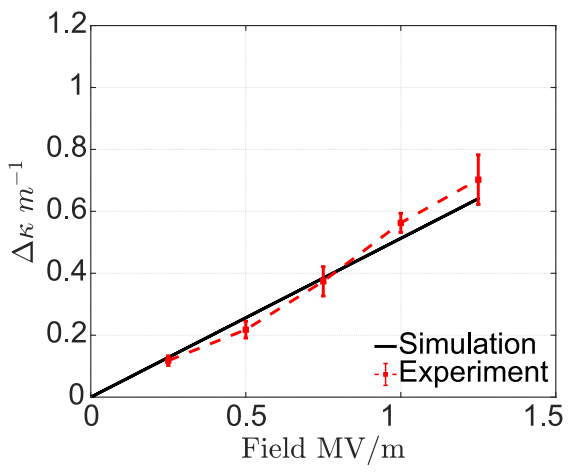

(e) $R=0.4$

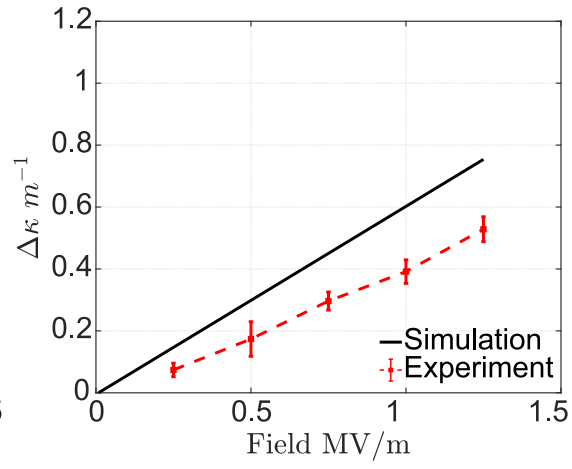

(c) $R=0.3175$

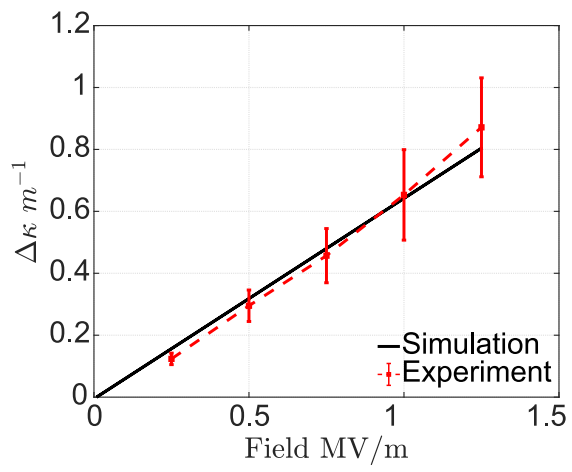

(f) $R=0.5$

Figure 6. Calibration of unimorph actuation under plane strain condition at low fields for different thickness ratios.

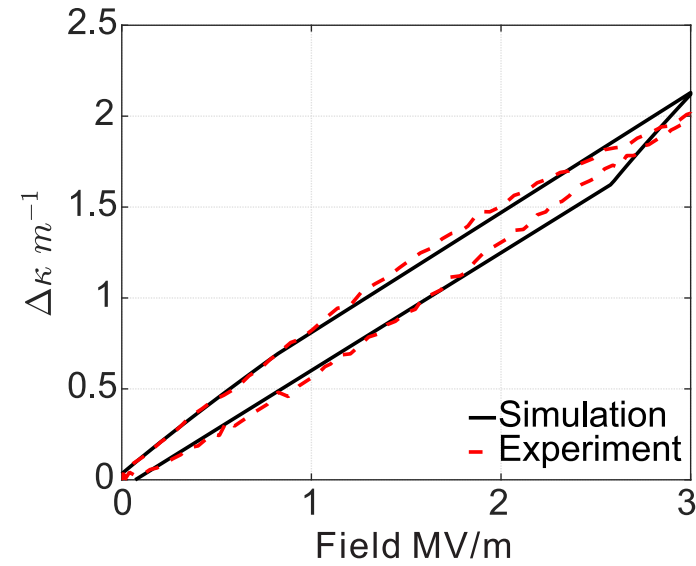

(a) $\mathrm{R}=0.5$

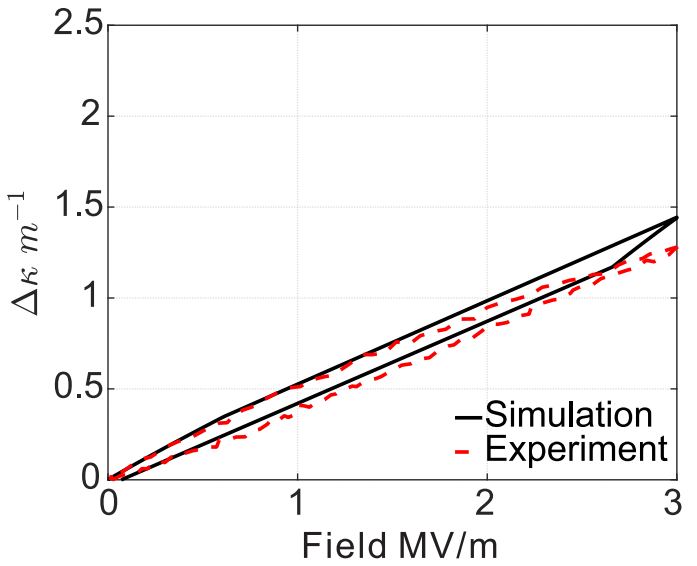

(b) $\mathrm{R}=0.33$

Figure 7. Calibration of unimorph actuation under plane strain condition at high fields. 


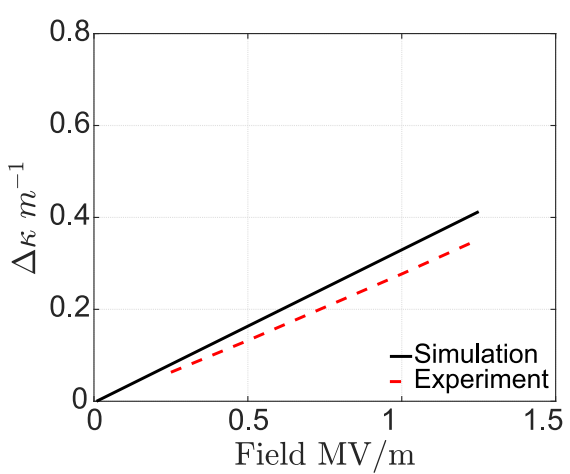

(a) $R=0.212$

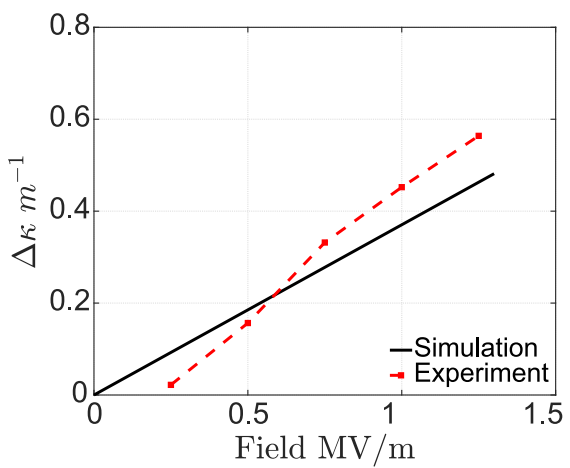

(d) $R=0.33$

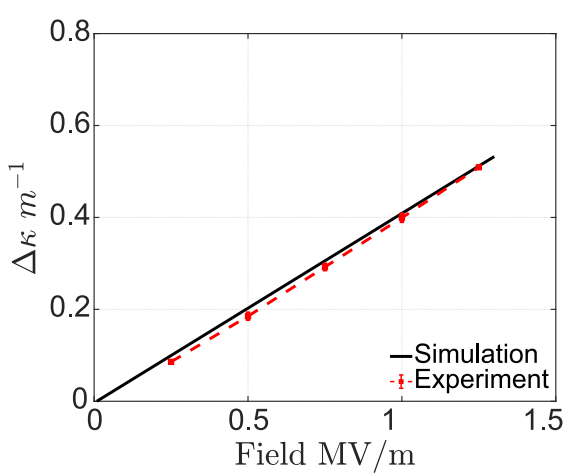

(b) $R=0.254$

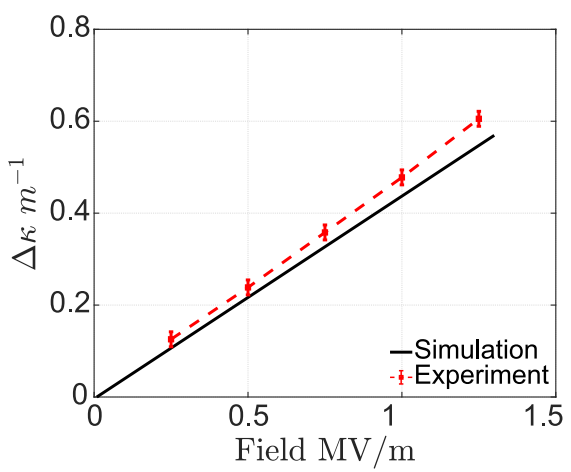

(e) $R=0.4$

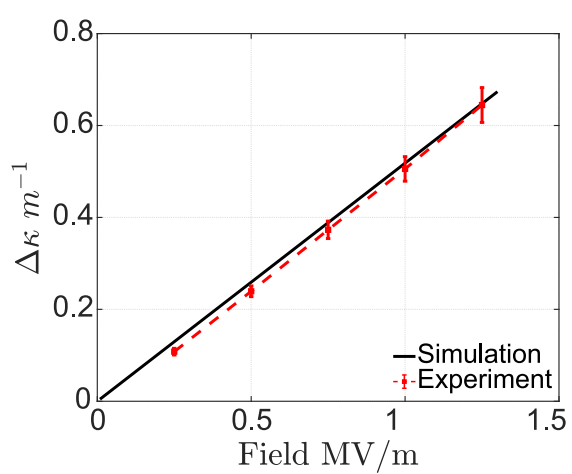

(c) $R=0.3175$

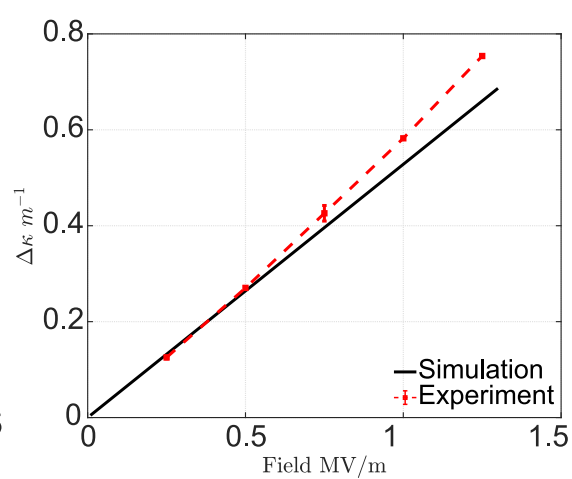

(f) $R=0.5$

Figure 8. Calibration of unimorph actuation under plane stress condition at low fields for different thickness ratios.

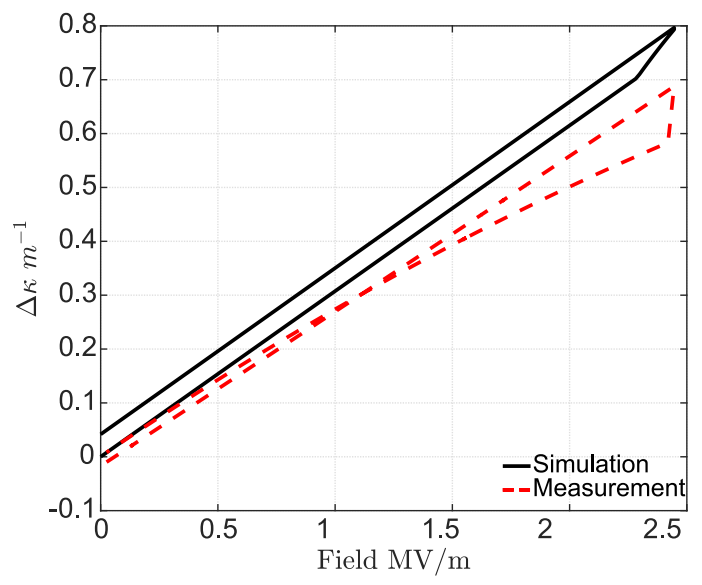

(a) $R=0.21$

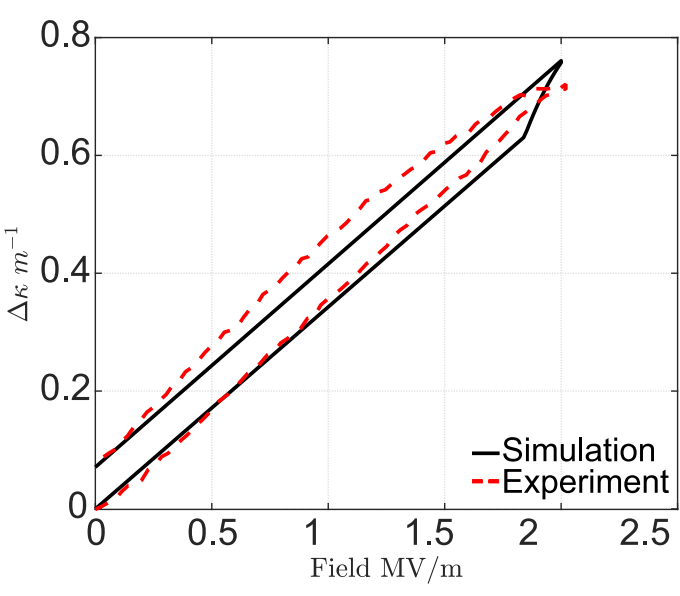

(b) $R=0.33$

Figure 9. Calibration of unimorph actuation under plane stress condition at high fields. 
Experimental curves in each figure represent the average of three sets of experiments. From figure 6 and 8 , it can be concluded that both the actuation condition and the thickness ratio $R$ greatly affect the electro-mechanical behavior of piezoelectric unimorph structures. For both actuation conditions, the magnitudes of $d_{31}$ and the nonlinearity of the curvature responses is proportional to $R$. Comparing the measurements of $\Delta K$ from the strip and the plate unimorph tests, when $R<0.4$ the differences between the results are within $10 \%$. However when $R>0.4$ and under high electric field $\mathcal{E}>\mathcal{E}_{0}$, the $\mathcal{E}-\Delta K$ curves deviate from linear relations. Explanations for such phenomena are two fold. First, the induced in-plane stress field magnitudes decrease as the thickness of piezoelectric layer becomes comparable with the substrate layer; for thin piezoelectric layers when $R$ is small, the 'clamping' effect from the substrate is more obvious, which inhibits a nonlinear response. This phenomenon was previously observed in ferroelectric membranes. ${ }^{33}$ Second, the absolute value of $d_{31}$ is higher under plane stress condition, which explains why plate unimorphs with thicker bonding layer still achieved similar $\Delta K$. The effects of in-plane stress field on $d_{31}$ are summarized at the end of this section.

\subsection{Combined calibration and optimal parameter selection}

So far, separate calibrations were performed on independent measurements. In order to find a set of model parameters that minimizes the NRMSD for total measurements, all sets of data points from section 4.2 and 4.3 were fitted in a single optimization process. This process is denoted as global fitting. Results from previous sections are referred to as local fittings. Furthermore, cross validations of NRMSD were carried on each measurement set with parameters from local fittings of different sources. The results for both global and local fitting, and cross validations are shown in table 2 .

Table 2. RMS error for different experiment sets

\begin{tabular}{ccccc}
\hline \hline & $\begin{array}{c}\text { Parameter } \\
\text { set 1 }\end{array}$ & $\begin{array}{c}\text { Parameter } \\
\text { set 2 }\end{array}$ & $\begin{array}{c}\text { Parameter } \\
\text { set 3 }\end{array}$ & $\begin{array}{c}\text { Parameter } \\
\text { set 4 }\end{array}$ \\
\hline $\begin{array}{c}\text { Experiment } \\
\text { set 1 }\end{array}$ & 8.5 & - & - & 38.8 \\
$\begin{array}{c}\text { Experiment } \\
\text { set 2 } \\
\text { Experiment } \\
\text { set 3 }\end{array}$ & 60 & 7.8 & 24.2 & 18.6 \\
Mean & 20 & 16.6 & 8.7 & 13.6 \\
\hline
\end{tabular}

${ }^{*}$ All values in $\%$.

In the table, diagonal entries correspond to local fittings informed by each parameter set, and achieve the lowest error. Off-diagonal terms store errors from cross checking calculations. For example, the entry of row 2 , column 3 is the error from estimation of plane stress actuation tests based on parameter set from plane strain calibration. Integration algorithm diverged for the cross check calculations in entries $(1,2)$ and $(1,3)$, so the mean error value were not calculated for this two columns. Comparing among all entries over the table, it can be concluded that to achieve a better fitness with a single measurement, the local approach will produce lower RMS (the diagonal terms). This suggests that for specific loading conditions, the parameter set obtained from local fitting will generate the closet predictions. Overall, parameter set 5 gives the lowest NRMSD, and was chosen as the optimal material parameters for the piezoelectric sheets studied in this research.

Table 3 extracts the $d_{31}$ values fitted from four sets of measurements, and the associated loading condition for each set of test. Clearly both electric and stress fields greatly affect the effective value of $d_{31}$ on the macroscopic level. In general, existence of electric field along poling direction of piezoelectric sheets enhances the in-plane piezoelectric effect, and $d_{31}$ depends on the magnitude of $\mathcal{E}$. Under moderate in-plane stress field, ferroelastically induced $d_{31}$ value is significantly lower than in the case of ferroelectric actuation (experiment set 2). When both fields exist in combination, the increase of in-plane stress level also resulted in a higher $d_{31}$ value. It is noticeable that these conclusions are obtained when the induced stress fields are tensile, i.e. $\sigma_{\alpha}>0$; the corresponding results under compressive fields remain to be tested. 
Table 3. Experiment conditions and piezoelectric constants

\begin{tabular}{lccc}
\hline \hline & $\begin{array}{c}\text { Experiment } \\
\text { set 1 }\end{array}$ & $\begin{array}{c}\text { Experiment } \\
\text { set 2 }\end{array}$ & $\begin{array}{c}\text { Experiment } \\
\text { set 3 }\end{array}$ \\
\hline $\mathcal{E}$ & $\sqrt{ }$ & $\sqrt{ }$ & $\sqrt{ }$ \\
$\sigma_{1}$ & - & $\sqrt{ }$ & $\sqrt{ }$ \\
$\sigma_{2}$ & - & $\sim 0.3 \sigma_{1}$ & $\sim \sigma_{1}$ \\
$d_{31} \mathrm{PC} / \mathrm{N}$ & -408.72 & -142.04 & -183.67 \\
\hline
\end{tabular}

\section{CONCLUSION}

The paper presented the analytical and experimental steps to calibrate a multi-axial, full field, rate independent phenomenological constitutive law for polycrystalline ferroelectric materials. The constitutive law was incorporated in a simplified, homogenized model for active structures actuation estimation, and an integration algorithm was implemented. Systematic experiments were carried on pre-poled thin PZT-5A sheets and unimorph structures manufactured from them. Material parameters incorporated in the constitutive law were fitted by minimizing the RMS error between experimental observations and corresponding estimations, and achieved

good agreement. Results of a comprehensive study on the relation between different fitting sources and model precision were also presented.

\section{ACKNOWLEDGMENTS}

The authors acknowledge helpful discussions with Professor Robert McMeeking and Professor Christopher Lynch.

\section{REFERENCES}

[1] A. J. Fleming, and K. K. Leang. "Design, modeling and control of nanopositioning systems." Switzerland: Springer International Publishing (2014).

[2] Md. A. Rahman, and A. Al. Mamun. "Nonlinearity Analysis, Modeling and Compensation in PZT Micro Actuator of Dual-stage Actuator System." IEEE/ASME Transactions on mechatronics 14.1, 21-31 (2009).

[3] APC International Ltd. "Piezoelectric ceramics: principles and applications, 2nd ed." APC International (2011).

[4] K. Patterson, N. Yamamoto, and S. Pellegrino. "Thin deformable mirrors for a reconfigurable space aperture. " 53rd AIAA/ASME/ASCE/AHS/ASC Structures, Structural Dynamics and Materials Conference (2012).

[5] J. Steeves. "Multilayer Active Shell Mirrors." Ph.D., California Institute of Technology (2015).

[6] C. M. Landis. "Non-linear constitutive modeling of ferroelectrics." Current Opinion in Solid State 8 Materials Science 8, 59-69 (2004).

[7] P. R. Potnis, N. T. Tsou, and J. E. Huber. "A review of domain modelling and domain imaging techniques in ferroelectric crystals." Materials 4.2, 417-496 (2011).

[8] J. Shieh, J. E. Huber, and N. A. Fleck. "An evaluation of switching criteria for ferroelectrics under stress and electric field." Acta Materialia 51(20), 6123-6137(2003).

[9] J. E. Huber "Electromechanical Models of Ferroelectric Materials." Ferroic Functional Materials Springer, Cham, 179-226(2018).

[10] Y. Su, and C. M. Landis. "Continuum thermodynamics of ferroelectric domain evolution: Theory, finite element implementation, and application to domain wall pinning. "Journal of the Mechanics and Physics of Solids 55(2), 280-305(2007).

[11] Schrade, D., et al. "Domain evolution in ferroelectric materials: A continuum phase field model and finite element implementation." Computer methods in applied mechanics and engineering 196(41), 4365-4374(2007). 
[12] A. Vidyasagar, W. L. Tan, and D. M. Kochmann. "Predicting the effective response of bulk polycrystalline ferroelectric ceramics via improved spectral phase field methods." Journal of the Mechanics and Physics of Solids (2017).

[13] S. C. Hwang, and R. M. McMeeking. "The predictions of switching in polycrystalline ferroelectric ceramics. "Ferroelectrics 207, 465-495 (1998).

[14] C. S. Lynch. "On the development of multiaxial phenomenological constitutive laws for ferroelectric ceramics." Journal of Intelligent Material System 9(7), 555-563 (1998).

[15] A. C. F. Cocks, and R. M. McMeeking. "A phenomenological constitutive law for the behaviour of ferroelectric ceramics." Ferroelectrics 228(1), 555-563 (1999).

[16] M. Kamlah, and C. Tsakmakis. "Phenomenological modeling of the non-linear electro-mechanical coupling in ferroelectrics." International Journal of Solids and Structures 36(5), 669-695 (1999).

[17] M. Kamlah, and C. Tsakmakis. "A thermodynamically and microscopically motivated constitutive model for piezoceramics." Computational Materials Science 28, 409-418(2003).

[18] C. M. Landis. "Fully coupled, multi-axial, symmetric constitutive laws for polycrystalline ferroelectric ceramics." Journal of the Mechanics and Physics of Solids 50(1), 127-152 (2002).

[19] C. M. Landis. "In-plane complex potentials for a special class of materials with degenerated piezoelectric properties." International Journal of Solids and Structures 41, 695-715 (2004).

[20] C. M. Landis, J. Wang, and J. Sheng. "Micro-electromechanical determination of the possible remnant strain and polarization states in polycrystalline ferroelectrics and the implications for phenomenologcial constitutive theories." Journal of intelligent material systems and structures 15, 513-525 (2004).

[21] S. Klinkel. "A thermodynamic consistent 1D model for ferroelastic and ferroelectric hysteresis effects in piezoceramics." Communications in Numerical Methods in Engineering 22(7), 727-739 (2006).

[22] S. Klinkel. "A phenomenological constitutive model for ferroelastic and ferroelectric hysteresis effects in ferroelectric ceramics." International Journal of Solids and Structures 43(22), 7197-7222 (2006).

[23] S. Maniprakash, R. Maniprakash, A. Menzel, and A. Arockiarajan. "Experimental investigation, modeling and simulation of rate-dependent response of 1-3 ferroelectric composites." Mechanics of Materials 94, 91-105 (2016).

[24] S. Maniprakash, R. Maniprakash, and A. Menzel. "A multi-surface model for ferroelectric ceramics application to cyclic electric loading with changing maximum amplitude." Philosophical Magazine 96(13), 1263-1284 (2016).

[25] E. F. Crawley, and K. B. Lazarus. "Use of piezoelectric actuators as elements of intelligent structures." AIAA journal 25.10, 1373-1385 (1987).

[26] E. F. Crawley, and K. B. Lazarus. "Induced strain actuation of isotropic and anisotropic plates." AIAA journal 29.6, 944-951 (1991).

[27] I. M. Daniel, et al. "Engineering mechanics of composite materials." New York : Oxford University Press (2006).

[28] D. L. DeVoe, and A. P. Pisano. "Modeling and optimal design of piezoelectric cantilever microactuators." Journal of Microelectromechanical Systems 6(3), 266-270 (1997).

[29] D. Damjanovic. "A morphotropic phase boundary system based on polarization rotation and polarization extension." Applied Physics Letters 97, 062906 (2010).

[30] J. Sinha. "Modified sawyer and tower circuit for the investigation of ferroelectric samples. "Journal of Scientific Instruments 42(9), 696(1965).

[31] P. Srinivasan, and S. M. Spearing. "Materials selection and design of microelectrothermal bimaterial actuators." Journal of Microelectromechanical Systems 16.2, 248-259 (2007).

[32] K. J. Yoon, K. H. Park, and S. K. Lee. et al. "Analytical design model for a piezo-composite unimorph actuator and its verification using lightweight piezo-composite curved actuators. "Smart Materials and Structures 13(3), 459 (2004).

[33] M. Wallace, R. L. Johnson-Wilke, et al. "In situ measurement of increased ferroelectric/ferroelastic domain wall motion in declamped tetragonal lead zirconate titanate thin films. " Journal of Applied Physics 117, 054103 (2015). 
[34] S. Stark, P. Neumeister, and H. Balke. "Some aspects of macroscopic phenomenological material models for ferroelectroelastic ceramics." International Journal of Solids and Structures 80, 359-367 (2016).

[35] T. Belytschko, et al. "Nonlinear finite elements for continua and structures." John wiley 6 sons (2013). 